\title{
New Device and Technology for High Speed Hydroforming
}

\author{
Matthias Hermes ${ }^{1, *}$, Aynur Keskin ${ }^{1}$, and Christoph Berlinger ${ }^{1}$
}

\author{
${ }^{1}$ Fachgebiet für Umformtechnik und Fügetechnik, Fachhochschule Südwestfalen, 59872 Meschede, Deutschland
}

\begin{abstract}
In the article a new production device is presented that combines the methodologies of high speed forming with conventional hydroforming. The result is a robust production system that can be easy controlled by the use of standard industrial components. The new system can be used as well for high automated production systems for mass production of small tubular and sheet metal parts as for the flexible manufacturing of workpieces with larger dimensions. The device is in principle a hydraulic driven high speed pressure generator that can be mounted directly on a hydroforming tool. Moreover, an additional drive for the axial feed of the tube is installed. In the paper the idea, the design of the device and the technical data is explained. Furthermore, the results of hydroforming experiments are presented and an overview on the specifications and the advantages of the new process is given. The strain rate is measured for an example workpiece and compared to the theoretical value. A first view on the forming limits is a further important topic of the paper. For the investigated copper tube, the forming limits are extended compared to a quasistatic hydroforming test.
\end{abstract}

Keywords: Hydroforming, Machine, Process

\section{Introduction}

Hydroforming is an important production technology since many years. Complex thin walled lightweight components for example for automotive applications can be produced in one process step on special production devices [1]. For smaller parts this technology is often inefficient because of high machine costs and the relatively high process time.

In recent years high speed forming methods were developed to produce sheet metal or tubular parts. Advantages of the high forming speed are among other things the extension of process limits and the production of workpieces with high geometrical complexity and accuracy in one process step $[2,3]$. Very well-known is the electromagnetic forming process that can be used for forming operations on tubular parts and sheet metal parts $[4,5]$. For bigger workpieces the energy of the electromagnetic devices is not sufficient. High speed forming technology then is driven by explosive materials or the detonation of gas mixtures. The problem of all high speed methods is the difficult control and automation of the process by standard control systems. Moreover, tools and production system components are not robust enough for industrial application and bigger production lots.

Obviously better control possibilities seem to have the plunger based systems because of the more conventional drive technology [6]. This technology is robust and components can be adjusted by conventional mechanical engineers. The problem of these processes is the limit in the production of complex large-surface parts because of the short pressure pulse. In the state of the art large surface parts are produced by quasi-static processes like deep drawing or conventional hydroforming. Thus a good innovation is the combination of the methodologies of high speed forming with conventional hydroforming. An interesting possible approach is the combination of quasistatic high pressure sheet metal hydroforming and pneumo-mechanical high speed forming on one and the same high speed forming machine [7].

In the following paper a new process and production device is shown that goes a similar way. It combines the methodologies of high speed forming with conventional tube hydroforming (THF). The result is a robust production system that is easy to control by standard industrial components. The new system can be used as well for highly automated production systems for mass production of smaller tubular and sheet metal parts as for the flexible manufacturing of workpieces with larger dimensions. The device is in principle a hydraulic driven high speed pressure generator that can be mounted directly on the hydroforming tool. When the idea was created a realistic approach was made how fast a hydraulic driven system could be. The idea was to fill the gap somewhere between conventional forming technology and the explosive forming or electromagnetic forming. To get a technological relevance the forming pressure should be as high as a conventional hydroforming process. In Fig. 1 (acc. to [8]) the planned range of forming speed and forming pressure for the new device is shown.

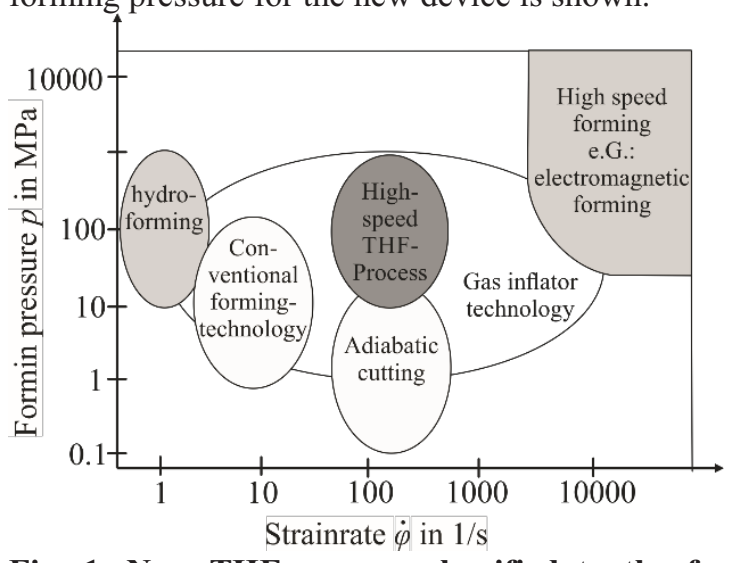

Fig. 1 New THF process classified to the forming speed according to [8]

\footnotetext{
* Corresponding author: hermes.matthias@fh-swf.de
} 


\section{Analysis of the process idea}

To develop a new device that fits to the process specifications of tube hydroforming (THF) the principle parameters has to be clarified. The THF-process has different parameters that are of interest. In Fig. 1 the different parameters are shown.

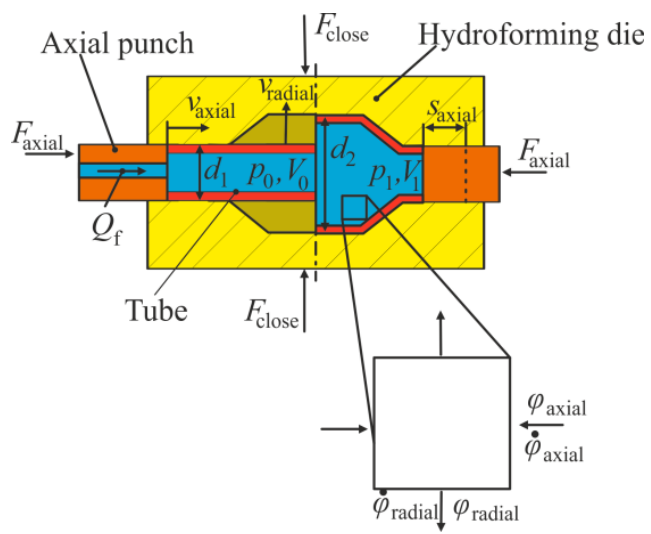

Fig. 2. Process principle and parameters of conventional and high speed THF process

The initial parameter $d_{1}$, the final diameter $d_{2}$ and the superposed axial feed $s_{\text {axial }}$ of the tube are of interest for quasi static THF process. Moreover, the filling pressure $p_{0}$ and the forming pressure $p_{1}$ have to be defined for a good working process. For high speed processes the parameters $v_{\text {axial }}$ und $v_{\text {radial }}$ are of additional interest because they define the forming speed during the process. The radial forming speed is influenced by the flow rate $Q_{\mathrm{f}}$. To reach extended forming limits and big diameters $d_{2}$ without cracks it is important to superpose an axial feed and efforts a high speed drive at the axial punch and a fast control system. Then it is possible to move the feed system with a high axial speed $v_{\text {axial }}$. Based on these thoughts an interesting process variant can be designed. For explanation in Fig. 2 a hypothetic THF process with low and with high speed (HSTHF) is shown.

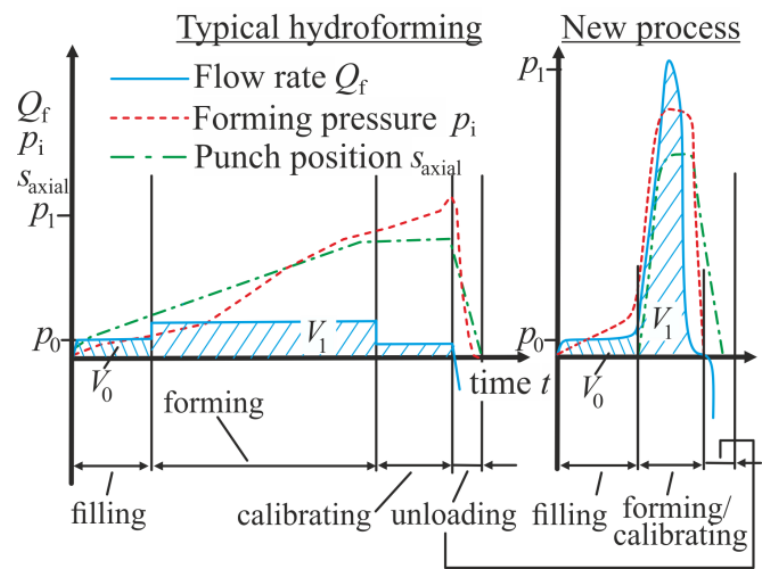

Fig. 3. Parameter variation in conventional THF and potential of HSTHF.

On the left side a typical hydroforming process diagram is shown. A relatively low flowrate fills the tube to $V_{0}$ and then $V_{1}$ is reached by further filling with a high pressure system. Simultaneously the axial feed pushes the tube into the die and the forming zone. The last optional step is calibrating the workpiece with high pressure and a small flow rate of fluid.

The diagram on the right shows a HSTHF process with superposed axial feed. The filling of $V_{0}$ is a quasi-static process, too. The second step shows the filling of $V_{1}$ by a high pressure pulse. The volume $V_{1}$ is filled during this pulse. This can be reached by a very high flow rate realized by a special high pressure pump that has a high speed drive. Moreover, the volume $V_{2}-V_{1}$ can be defined exactly by the pump and an overloading in principle is not necessary because the calibration step and the main forming step is superposed in one small time step. Moreover, it is thinkable that by the high short pressure pulse shockwaves are superposed with the simple pumping of the fluid. This can influence the forming process as well. Additionally, the axial feed of the tube is realized by a high speed drive and can be started during the same time step or earlier. Compared to conventional THF there are some differences that occurs an interesting potential:

1. The short pressure pulse and the high flow rate make high forming speeds possible. So the typical extended forming limits because of high strain rates are thinkable. Moreover, the superposed axial feed makes it possible to reduce the thinning of the tube wall thickness and enhance the forming limits further.

2. The high forming speed achieves a high radial speed $v_{\text {radial }}$ and so a high accuracy and surface quality can be achieved. Moreover, complex geometries with small radii can be formed and calibrated.

3. The short process time has the potential to use the process in mass production and can be coupled in process chains with other fast production processes with short circle times.

4. The closing force $F_{\text {close }}$ can be reduced by the short pressure pulse and the defined volume of fluid $V_{2}-V_{1}$. That allows locking the die for example by mass inertia.

5. The defined volume of forming fluid that is pumped into the workpiece allows producing big surface parts, because there is more than a short pressure pulse like in other high speed forming methods.

6. Sealing is often a problem at fluid based forming processes. Because of the high flow rate small leakage means no eminent reduction of the pressure maximum because of the dependency of pressure and flow rate.

7. The short pressure pulse gives the potential of shock waves superposed with the high flowrate that can enhance the forming process.

These potentials have to be investigated and achieved by an experimental device that was developed at the laboratory. The device is designed very flexible, so it can realize a big range of parameter combinations and process curves according to Fig. 2. The design and the specifications are shown in chapter 2 . 


\section{Concept and design of the device}

The list of requirements for the device includes the following main points:

- Realization of a high flow rate,

- Realization of a high and short pressure pulse,

- Realization of a high speed drive for axial feeding of the tube,

- the use of conventional hydraulic components and a conventional PLC,

- and a design that makes a flexible adjusting of all parameters and further developments possible

Considering these requirements, a flexible testing device for HSHF was designed [9]. The functional scheme of the new device is shown in Fig. 3.

The high flow rate is based on at least one hydraulic accumulator that is loaded with a standard hydraulic pump with relatively small flow rate. In the realized device two accumulators were used. The accumulator characteristic can be adjusted by a $\mathrm{N}_{2}$ bottle and a high pressure gas valve. In a steel block a cartridge seat valve NG 46 is mounted. This size is selected to reduce pressure losses and to reach a high flow rate in the primary side of the device.

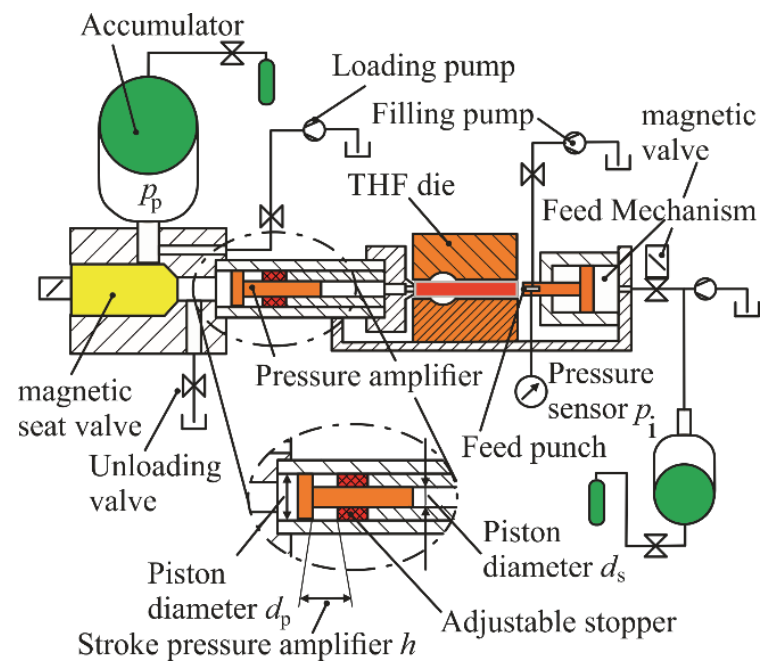

Fig. 4. Functional scheme of the forming device

To start the process the seat valve is opened by the control system (Siemens S7) and hydraulic fluid runs into a pressure amplifier. The pressure pulse pushes the piston onto an adjustable stopper that defines the stroke. By that the volume of forming fluid is pumped on the secondary side of the system into the THF-die. The pressure amplifier raises the pressure of the conventional hydraulic pressure to the forming pressure and separates the hydraulic oil from the water based forming fluid. The flow rate is of course reduced. The transformation ratio of the amplifier can be adjusted by exchangeable piston/sleeve inserts. The primary pressure pump (loading pump) is a conventional hydraulic pump that defines the pressure in the low pressure side of the pressure amplifier $\left(p_{\mathrm{p}}=30\right.$
$\mathrm{MPa}$ ). At the moment two variants of amplifiers are possible to mount in the system. The first delivers a forming pressure of $250 \mathrm{MPa}$ but has because of a smaller bore on the secondary side a lower flow rate. The second amplifier has a bigger flow rate and a maximum pressure of $140 \mathrm{MPa}$. The primary flow rate is defined by the accumulators. They have each the potential of $30 \mathrm{l} / \mathrm{s}$.

On the other side of the THF-die an axial feed system is installed. This has a hydraulic drive system with an accumulator. By that the axial feed can be driven also with higher speed values. The start signal for the installed magnetic valve is controlled by the $\mathrm{S} 7$ also. The start time can be adjusted in steps of $0.05 \mathrm{sec}$. So the valve can be opened simultaneously, before or after the process start of the forming process.

The feed punch is prepared for the filling system that fills the secondary system and the tube with the forming fluid. The filling pump has enough pressure to push the pressure amplifier in the start position. Moreover, it is possible to pre-form the workpiece quasi-static and to use the system for conventional THF ( $\left.\mathrm{p}_{\max }=360 \mathrm{MPa}\right)$. The device and its control system allow the investigation of a wide range of different HSTHF process variants in addition to the variants shown in Fig. 2. The design of the device is very flexible and is continuously extendable. In Table 1 actual specifications are listed.

Table 1. Specifications of the devive (status quo).

\begin{tabular}{|c|c|}
\hline Specification & Potential \\
\hline Filling pump pressure $p_{0, \max }$ & $0 \ldots 360 \mathrm{MPa}$ \\
\hline Primary pressure max $p_{\mathrm{p}, \max }$ & $30 \mathrm{Mpa}$ \\
\hline $\begin{array}{c}\text { Secondary pressure } \\
\text { forming pressure, depends } \\
\text { on plunger diameter) max. } \\
p_{\mathrm{i}, \max }\end{array}$ & $250(140) \mathrm{MPa}$ \\
\hline $\begin{array}{c}Q_{\mathrm{p}, \max } \\
\text { Primary flow rate } \\
Q_{\mathrm{f}, \max }\end{array}$ & approx. $601 / \mathrm{s}$ \\
\hline $\begin{array}{c}\text { Secondary flow rate max. } \\
\text { approx. } 7,5(11,5) 1 / \mathrm{s}\end{array}$ \\
\hline $\begin{array}{c}\text { Force of the axial feed } \\
\text { punch max. } F_{\text {axial, max }}\end{array}$ & $7,8 \mathrm{kN}$ \\
\hline $\begin{array}{c}\text { Axial feed speed max } \\
v_{\text {axial,max }}\end{array}$ & approx. $3,5 \mathrm{~m} / \mathrm{s}$ \\
\hline
\end{tabular}

In Fig. 4 the experimental setup is shown in reality. The red painted high pressure device is coupled with flexible pipes and control cables. This makes it possible to mount the device on other production systems for example stamping dies or special tube forming machines. The device is continuously developed. An important aim of the project is getting knowledge for future industrial machines. 


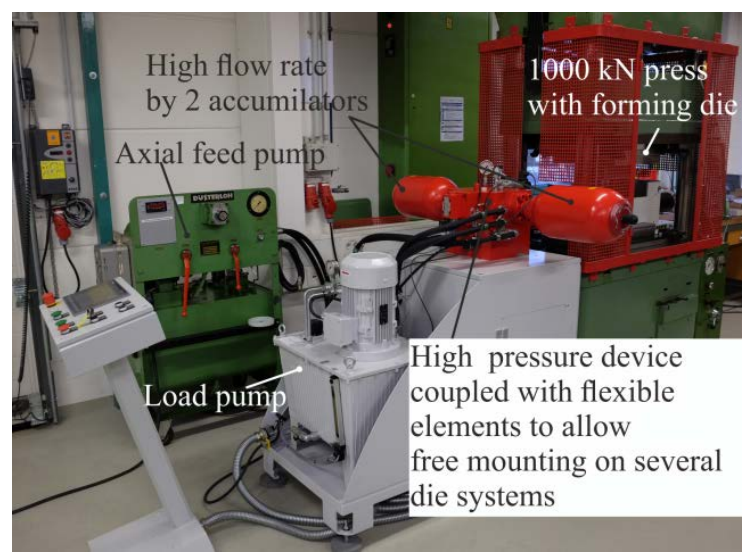

Fig. 5. Photo of the experimental setup

\section{First results}

To get more information about the process potential the theoretical values were investigated by experiments.

In Fig 5 the pressure rise of the 140-MPa-amplifier is shown. It can be said that the pressure amplifier generates the expected pressure values. The rise is more interesting with view on the forming speed. At the current experiments the rise is generated in time steps smaller than $20 \mathrm{~ms}$. For future developments this should be shorter. The reason lies in the opening time of the seat valve of $40 \mathrm{~ms}$. The aim is to reach 1 or $2 \mathrm{~ms}$ by changing the seat valve to a faster one. In the described concept in Fig. 2 the pressure peak is short and falls after reaching the maximum. This wasn't tested till now. In principle it is possible by unloading the primary pressure by the unloading valve (Fig. 3).

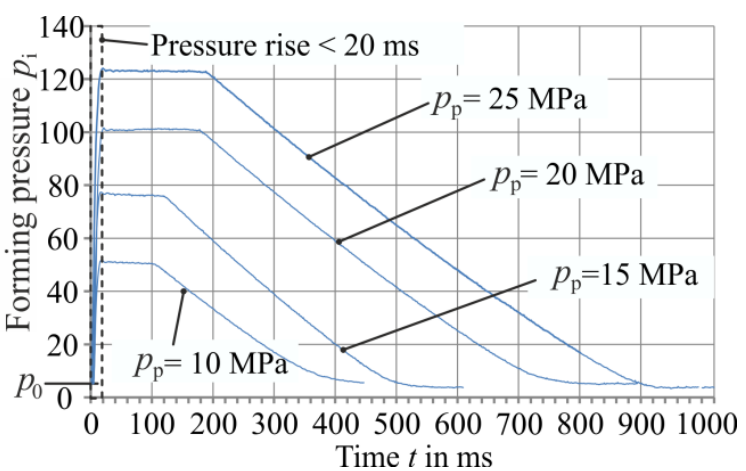

Fig. 6. Pressure rise and fall at actual status of the device.

More interesting is the radial and axial forming speed $v_{\text {axial }}$ and $v_{\text {radial }}$. Till now some investigations regarding $v_{\text {rad }}$ were done. The theoretical value can be calculated by the time $t_{\mathrm{f}}$ that is needed to fill the workpiece geometrie with the volume $V_{1}+V_{2}$ during the forming process. This can be calculated with the theoretical maximum flow rate of the device:

$$
t_{f}=\frac{V_{1}+V_{2}}{Q_{f, \max }}
$$

The average strainrate assuming a pure radial forming can be estimated with

$$
\dot{\varphi} \approx \frac{\ln \frac{d_{2}}{d_{1}}}{t_{f}},
$$

where $d_{1}$ und $d_{2}$ depend of the die design. To prove this theory experiments were made with a die that was mounted into the device. A copper tube $d_{1}=22 \times 1 \mathrm{~mm}$ was used as specimen. Special probes where developed to measure by contact the time between starting and the end of the expansion. The experimental setup and the result are shown in Fig. 6.

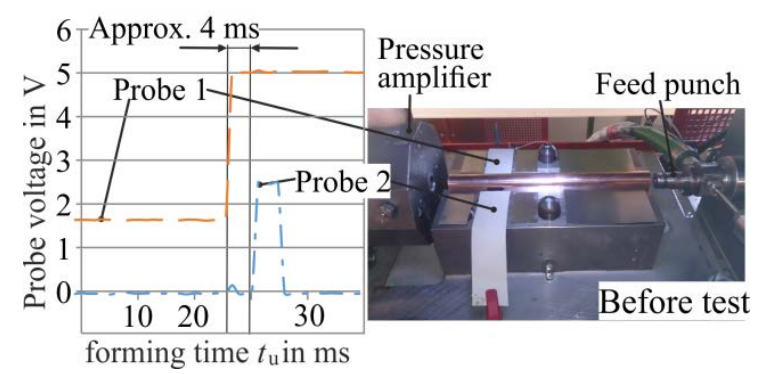

Fig. 7. Speed measurement with conductive probes

The probes where made of thin copper sheets and coated with isolation paint. The probes where positioned between the die halves and conducted to a fast measurement system that determines the time of contact between the tube wall and the probe 1 and probe 2 by a voltage signal. The time between the contacts is about $4 \mathrm{~ms}$ with maximum loading of the device and using the $250 \mathrm{MPa}$ pressure amplifiers. With (1) and (2) a strain rate of approx. $70 \mathrm{~s}^{-1}$ could be calculated. This fits very well to the theoretical value. It has to be said that this is not very fast for ha high speed process but even faster than quasi static processes. The influence of additional axial feed on the forming speed is not yet tested. The 140-MPaamplifier can approximately deliver the double forming speed. Interesting is that the relatively slow seat valve does not influence the process. In future the forming speed is planned be raised by further development of the device. A second interesting experiment is shown in Fig. 7.

Here the influence of the axial feed combined with the high forming speed was tested. The results are shown at the example of copper tubes $\left(d_{1}=22 \times 1 \mathrm{~mm}\right.$, material $\mathrm{R} 250 \mathrm{HH})$ that were expanded to $d_{2}=33 \mathrm{~mm}$ into a forming die. For lubrication industrial petroleum jelly was used. For comparison a tests under quasi static forming were done and failed by a typical tangential crack in the tube wall. The next test group were made under dynamic forming and shows no crack but a stronger thinning. Moreover, in some areas on the surface the material localizes and the stadium before cracking could be detected. The thinning was analyzed by cutting the tube in halve. The tubes were $9 \mathrm{~mm}$ shorter so the forming comes not only out of the wall thickness. The second test where superposed by an axial feed with $s_{\text {axial }}=17 \mathrm{~mm}$. The workpieces has a little less thinning in the expanded area. So even in this early state of the investigations it can be said that the axial feed has a positive effect on the HSTHF process. 


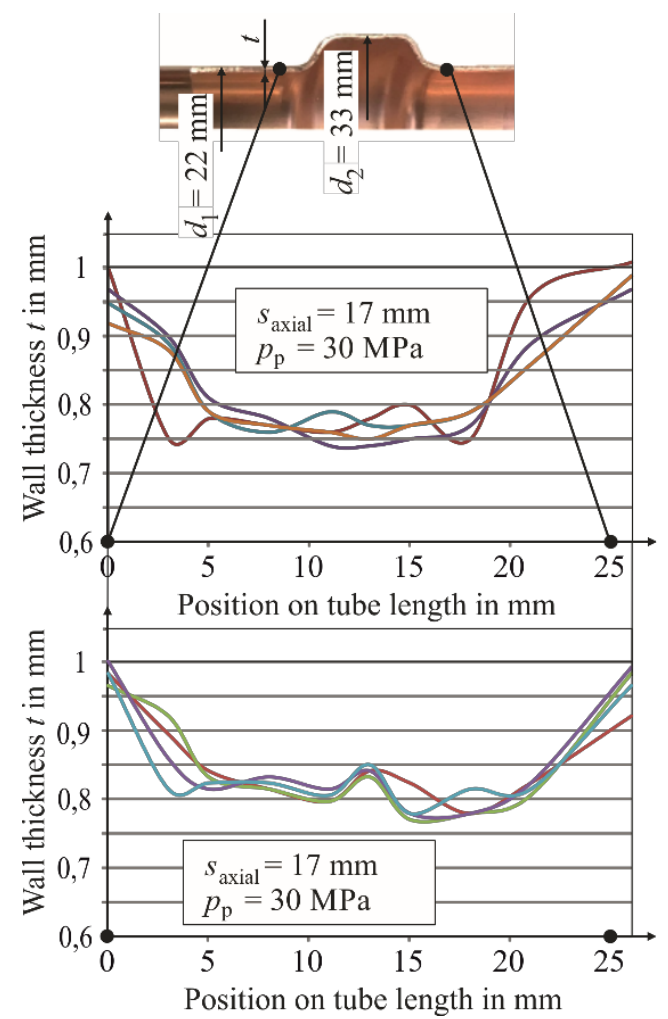

Fig. 8. Wall thickness deviation over the longitudinal axis

\section{Conclusion and outlook}

First step of the work was the design of a flexible high speed hydroforming device that uses conventional hydraulic and control components. The technical function of the system was tested and showed a good performance. First aim of the investigations with the device concentrated on the function of the machine. Input output relation of the parameters fit to the requirements. Investigations regarding the strain rates showed that parameters can be controlled with a good precision. Further tests were made with copper tubes. They are expanded from $22 \mathrm{~mm}$ on $33 \mathrm{~mm}$, showed enhanced process limits in comparison to the quasi static THF process. Moreover, it could be seen that the installed high speed axial feed system reduces thinning of the wall thickness.

The device was designed to make future upgrading easy possible. Aim of the next work is to extend values of forming speed, flow rate and pressure pulse. The Pressure pulse should be shortened.

Furthermore, aim of future process investigations is the variation of workpiece materials. Moreover, the forming limits, the variation of the parameters forming time and the effect of axial feed will be investigated.

At the moment a FE-model is developed to investigate process mechanisms in detail.

Further activities in the field of applied research are the integration of the device in coupled processes and in special forming machines to analyze the possibilities of process automation. Furthermore, the superposition of other production operations for example high speed blanking will be investigated.
The project is supported by transfluid Maschinenbau $\mathrm{GmbH}$, Schmallenberg, Germany. The company has helped to build the device and supports the experiments with raw material and tools.

\section{References}

1. R. Neugebauer, Hydro-Umformung. ISBN-13 978-3-540-21171-6, Springer 2007

2. X. Hu, G.S. Daehn, Acta mater. 44, 1021 (1996)

3. V. Balanethiram, X. Hu, M. Altynova, G.S. Daehn,. Journal of Materials Processing Technology, 45, 595, (1994)

4. C. Beerwald, Grundlagen der Prozessauslegung und -gestaltung bei der elektromagnetischen Umformung. Dissertation, Dortmund (2004)

5. V. Psyk, D. Risch, B. L. Kinsey, A. E. Tekkaya, M. Kleiner, Journal of Materials Processing Technology, 211, 5, 787, (2011),

6. W. Homberg, E. Djakow, O. Akst, ICHSF 2012, Dortmund, Germany, 2012

7. E. Djakow, W. Homberg, D. Tabakajew, ICHSF 2016, Dortmund, Germany, 2016

8. H. Hoffmann, R. Neugebauer, G. Spur, Handbuch Umformen. ISBN: 978-3-446-427785, Hanser 2012

9. M. Hermes, M. Böhmer, European patent application, EP 17400044, (2017) 